\title{
A modified form of the polar model of crystals
}

\author{
L.Didukh \\ Ternopil State Technical University, Department of Physics, \\ 56 Rus'ka St., UA-282001 Ternopil, Ukraine
}

Received May 22, 1998

\begin{abstract}
A model for the description of materials with narrow energy bands is proposed. It is shown that in narrow-band materials an electron-hole symmetry is absent in contrast to the Hubbard model. In this paper a metal-insulator transition is studied. The obtained results are compared with the experimental data for narrow-band materials. Some specific narrow-band effects are discussed.
\end{abstract}

Key words: narrow energy bands, model Hamiltonian, correlated hopping, electron-hole asymmetry

PACS: $71.28 .+d, 71.27 .+a, 71.10 . F d, 71.30 .+h$

\section{Introduction}

1. The fact that the amasing properties of narrow-band systems (for example, oxides, sulphides and selenides of transition metals) are caused by the electronelectron interactions is generally accepted nowadays. But in spite of the great number of papers concerning this problem, it is still an actual task for condensed matter physics to develop a consistent theory of narrow-band systems. During the last years a range of problems were connected with corellations, and the necessity of an investigation of narrow-band systems has greatly increased because of the discovery of high- $T_{c}$ superconductors.

The arising problems can be divided into three groups: 1) construction of the narrow-band systems models by using adequate Hamiltonians; 2) elaboration of the effective mathematical methods to study the model Hamiltonians; 3) construction of a consistent theory of correlation effects and explanation of the peculiarities of physical properties in narrow-band systems.

Problems 1 and 2 were considered and partially solved by S.Schubin and S.Wonsowsky in their famous theory of the polar model [1]. In that theory the polar model Hamiltonian and its "configurational" representation was proposed. The polar model proves to be very meaningful owing to the euristic value of the "config- 
urational" description idea (basical for the model treatment of $3 d$-compounds [2].) Within the framework of the polar model a criterion of the metal-insulator transition (MIT) was formulated for the first time; explanation of the fractional atom momentum in transition $3 d$-metals and a hypothesis of the possibility of charge ordering were proposed; a possibility of the existence of gapless semiconductors and a superexchange interaction were predicted. The commonly used Hubbard model is a partial case of the polar model [3].

However, it was proved that a direct use of the polar model (in the traditional form [1]) for the solution of the above mentioned problems was not effective in many cases.

Firstly, transition from the second quantization Hamiltonian in terms of electron operators to its representation in terms of Schubin-Wonsowsky operators is realized by the substitution of some combinations of electron operators by the combinations of Shubin-Wonsowsky operators with the same action on the wave function. Such transition is bulky and difficult even for $s$-band (see, for example, $[4])$.

Secondly, approximations underlying the mathematical treatment of the polar model are uncontrollable (first of all, the postulation of the Bose-type commutation rules for current excitation operators).

2. The polar model theory was developed in two ways. The first is connected with developing the methods of effective mathematical treatment of the initial polar model Hamiltonian in the electron representation. The fundamental results in this way belong to N.Bogolyubov [5]. He proposed the effective Hamiltonian method which took into account the high energy electron states with the help of a special form of the perturbation theory. This method is one of the most consistent approaches to study the exchange interactions in magnetic insulators [6]. The use of the configurational representation of the polar model (polar and homeopolar states) proved to be helpful for the interpretation of the obtained results and control of the performed calculations (sometimes very bulky).

The second way is based on the immediate use of configurational representations. This approach is effective for the investigation of the peculiarities of narrowband systems, insulators and semiconductors, on the one hand, and metals and materials in which MIT is caused by external influences, on the other one.

An important achievement in this way was obtained in the works of researchers of the Lviv branch in the theory of solids. Among them were the pioneer works [79] by A.Yu.Glauberman, V.V.Vladimirov and I.V.Stasyuk. In this way important problems of the polar model of non-metallic crystals (the problem of the construction of model Hamiltonians in terms of the site elementary excitations and the problem of commutation rules for the site elementary excitations operators) were solved.

3. A wide use of the configurational forms of model Hamiltonians in considering physical properties of narrow-band materials is connected with the paper by J.Hubbard [10] in which $X_{i}^{k l}$-operators were introduced and papers $[11,12]$ where the relations between the electron and transition operators were first established and 
an effective form of the perturbation theory was proposed. It has been shown that the proper identification of the Schubin-Wonsowsky operators and transition operators (the Hubbard operators) leads to formal equivalence of the traditional form of the polar model and its modern representations in terms of transition operators. It was also shown that

$$
X_{i}^{k l}=\alpha_{i k}^{+} \alpha_{i l}
$$

where $\alpha_{i k}^{+}, \alpha_{i l}$ are Schubin-Wonsowsky operators of creation and destruction of $|k\rangle$ and $|l\rangle$ states, respectively, on $i$-site.

An expediency of using $X_{i}^{k l}$ - or $\alpha_{i \nu}^{+} \alpha_{i \mu^{-}}$representation is determined by the requirements of the considered problem. In calculations employing the diagrammatic Green functions technique or the perturbation theory it is convenient to use $X_{i}^{k l}$-representation of the Hamiltonian [13], whereas in problems using the approximate second quantization method (e.g. to study MIT using the mean-field approximation (MFA) in the generalized Hartree-Fock approximation [14]) $\alpha_{i \nu}^{+} \alpha_{i \mu^{-}}$ representation is more convenient. The $b$ - $c$-representation (see Sect. 2) can also be useful.

4. The representations of the model narrow-band Hamiltonians in terms of $X_{i}^{k l}$-, $b$-c- or $\alpha_{i \nu}^{+} \alpha_{i \mu}$-operators are helpful for understanding the physics of the correlation effects in narrow energy bands and explanation of the physical properties of narrow-band materials. These representations are convenient from the point of view of the mathematical treatment of models.

Below a consistent form of the polar model of narrow-band materials is proposed and the consequences of this model are considered.

\section{The Hamiltonian}

Hamiltonian of the system of $s$-electrons in the Wannier representation is written as

$$
\begin{aligned}
H & =-\mu \sum_{i \sigma} a_{i \sigma}^{+} a_{i \sigma}+\sum_{i j \sigma}^{\prime} t(i j) a_{i \sigma}^{+} a_{j \sigma}+ \\
& +\frac{1}{2} \sum_{\substack{i j k l \\
\sigma, \sigma^{\prime}}} J(i j k l) a_{i \sigma}^{+} a_{j \sigma^{\prime}} a_{l \sigma^{\prime}} a_{k \sigma}
\end{aligned}
$$

where $a_{i \sigma}^{+}, a_{i \sigma}$ are creation and destruction operators of an electron on site $i$, $\sigma=\uparrow, \downarrow, \mu$ is a chemical potential, $n_{i \sigma}=a_{i \sigma}^{+} a_{i \sigma}$,

$$
\begin{aligned}
& t(i j)=\int \phi^{*}\left(\mathbf{r}-\mathbf{R}_{i}\right) \sum_{n} V\left(\mathbf{r}-\mathbf{R}_{n}\right) \phi\left(\mathbf{r}-\mathbf{R}_{j}\right) \mathrm{d} \mathbf{r}, \\
& J(i j k l)=\iint \phi^{*}\left(\mathbf{r}-\mathbf{R}_{i}\right) \phi\left(\mathbf{r}-\mathbf{R}_{k}\right) \frac{e^{2}}{\left|\mathbf{r}-\mathbf{r}^{\prime}\right|} \times \\
& \times \phi^{*}\left(\mathbf{r}^{\prime}-\mathbf{R}_{j}\right) \phi\left(\mathbf{r}^{\prime}-\mathbf{R}_{l}\right) \mathrm{d} \mathbf{r} \mathrm{d} \mathbf{r}^{\prime},
\end{aligned}
$$


are the matrix elements which describe the hoppings of electrons between the nearest-neighbour sites of the lattice due to the electron-ion $\left(V\left(\mathbf{r}-\mathbf{R}_{i}\right)\right.$ is the potential energy of an electron interacting with an ion at $i$-site) and electronelectron interactions. The prime at the second sum in equation (2.1) signifies that $i \neq j$.

Narrow energy bands allow one to simplify Hamiltonian (2.1). Here wave functions are close to atomic $3 d$-functions (their overlapping decreases quickly with the increase of the inter-atomic spacing), so matrix elements $t(i j)$ and $J(i j k l)$ can be estimated from the degree of overlapping. Thus, quantities $J(i i i i)$ and $J(i k i k)$ will be of the zero order, $J(i i i j), J(i j k j)$ - of the first order (as $t(i j)), J(i j k l)$ at $i \neq k, j \neq l$ - of the second order (immediate estimation of $J(i j k l)$ is given in paper [3]). In accordance with this we limit ourselves to considering in Hamiltonian (2.1) the matrix elements $J(i i i i)=U, J(i j i j)=V(i j)$ (i and $j$ are nearest neighbours), $J(i i i j)=T(i j), J(i j k j)(k \neq i, k \neq j), J(i j j i)=J(i j)$; taking into account the quantity of the second order $J(i j)$ is, in principle, necessary to describe ferromagnetism in this model in the Mott-Hubbard insulator state. Then,

$$
\begin{aligned}
H= & -\mu \sum_{i \sigma} a_{i \sigma}^{+} a_{i \sigma}+ \\
& +\sum_{i j \sigma}^{\prime} a_{i \sigma}^{+}\left(t(i j)+\sum_{k} J(i k j k) n_{k}\right) a_{j \sigma}+U \sum_{i} n_{i \uparrow} n_{i \downarrow}+ \\
& +\frac{1}{2} \sum_{i j \sigma \sigma^{\prime}}^{\prime} J(i j) a_{i \sigma}^{+} a_{j \sigma^{\prime}}^{+} a_{i \sigma^{\prime}} a_{j \sigma}+\frac{1}{2} \sum_{i j \sigma \sigma^{\prime}}^{\prime} V(i j) n_{i \sigma} n_{j \sigma^{\prime}},
\end{aligned}
$$

where $n_{i}=n_{i \uparrow}+n_{i \downarrow}$.

In Hamiltonian (2.1) we rewrite the sum $\sum_{i j \sigma k}^{\prime} J(i k j k) a_{i \sigma}^{+} n_{k} a_{j \sigma}$ in the form

$$
\sum_{i j \sigma}^{\prime} \sum_{\substack{k \neq i \\ k \neq j}} J(i k j k) a_{i \sigma}^{+} n_{k} a_{j \sigma}+\sum_{i j \sigma}^{\prime}\left(J(i i i j) a_{i \sigma}^{+} a_{j \sigma} n_{i \bar{\sigma}}+\text { h.c. }\right)
$$

$(\bar{\sigma}$ denotes spin projection which is opposite to $\sigma$ ). We suppose (as in papers $[14$, 15]) that

$$
\sum_{i j \sigma}^{\prime} \sum_{\substack{k \neq i \\ k \neq j}} J(i k j k) a_{i \sigma}^{+} n_{k} a_{j \sigma}=n \sum_{\substack{k \neq i \\ k \neq j}} J(i k j k) \sum_{i j \sigma}^{\prime} a_{i \sigma}^{+} a_{j \sigma}
$$

with $n=\left\langle n_{i \uparrow}+n_{i \downarrow}\right\rangle$ (sites $i$ and $j$ are nearest neighbours). It should be noted that this supposition is exact in the homeopolar limit $\left(n_{i}=1\right)$.

Thus, Hamiltonian (2.4) takes the following form

$$
\begin{aligned}
H= & -\mu \sum_{i \sigma} a_{i \sigma}^{+} a_{i \sigma}+\sum_{i j \sigma}^{\prime} t_{i j}(n) a_{i \sigma}^{+} a_{j \sigma}+ \\
& +\sum_{i j \sigma}^{\prime}\left(T(i j) a_{i \sigma}^{+} a_{j \sigma} n_{i \bar{\sigma}}+\text { h.c. }\right)+U \sum_{i} n_{i \uparrow} n_{i \downarrow}+ \\
& +\frac{1}{2} \sum_{i j \sigma \sigma^{\prime}}^{\prime} J(i j) a_{i \sigma}^{+} a_{j \sigma^{\prime}}^{+} a_{i \sigma^{\prime}} a_{j \sigma}+\frac{1}{2} \sum_{i j \sigma \sigma^{\prime}}^{\prime} V(i j) n_{i \sigma} n_{j \sigma^{\prime}}
\end{aligned}
$$


where

$$
t_{i j}(n)=t(i j)+n \sum_{\substack{k \neq i \\ k \neq j}} J(i k j k)
$$

is an effective hopping integral between the nearest neighbours.

Neglecting all the matrix elements in (2.6) except for $t(i j)$ and $J(i i i i)$ we obtain the Hubbard Hamiltonian.

Transition from the general form of Hamiltonian (2.6) to the Hubbard Hamiltonian, i.e. taking into account only the intra-atomic Coulomb repulsion, is usually argued by the smallness of quantities $J(i i i j), J(i k j k), J(i j j i)$ and $J(i j i j)$ in comparison with $J($ iiii). However, taking into account these matrix elements can be of principal importance from the points of view of both the construction of the correlation effects theory in materials with narrow energy bands and the interpretation of physical properties of these materials [13, 15-17].

The neglecting of the inter-atomic exchange interaction is justified by the smallness of $J(i j)$ in comparison with $U$ and hopping integral $t(i j)$, on the one hand, and a possibility of the ferromagnetic ordering stabilization in the narrow energy band (NEB) in consequence of the "translational" mechanism of the exchange, on the other hand. Without the consideration of a possibility of ferromagnetism realization in the one-band Hubbard model it should be noted that in NEB a contribution of the translational part of energy in the total system energy can be smaller than the contribution of energy of the inter-atomic exchange interaction (in spite of the fact that $|t(i j)| \gg J(i j)$ ). Really, in a partially filled NEB (for $U \gg|t(i j)|$ ) the contribution of the translational part of the ground state energy is $\sim n \delta w(\delta$ is a degree of deviation from half-filling, $n$ is the average number of electrons on the site, $2 w$ is the bandwidth) [16], and the contribution of the exchange interaction into the ground state energy is $\sim z n^{2} J$ ( $J$ is an exchange integral between the nearest neighbours, $z$ is the number of the nearest neighbours to the site). It is clear, that in the NEB close to half-filling $(\delta \rightarrow 0)$ the contribution of energy of the inter-atomic exchange interaction in the total system energy will be the biggest. In particular, in non-doped Mott-Hubbard ferromagnets the magnetic ordering is stabilized by the inter-atomic exchange interaction only.

Taking into account the inter-atomic Coulomb interaction is of principal importance to understand the character of the charge ordering in materials with NEB.

Finally, the neglecting of the correlated hopping (2.5) is justified by the estimation of matrix elements [3]. However, matrix elements $J(i k j k)$ are hopping integrals. Thus, taking into account (2.5) leads to renormalization of the translational processes describing the band part of Hamiltonian (2.6). Here $t(i j), T(i j)$, $J(i k j k)$ are quantities of the same order.

If the direct exchange interaction and the inter-atomic Coulomb repulsion can be taken into account by the respective renormalization of the chemical potential (when ferromagnetic and charge orderings are absent), then Hamiltonian (2.6) 
takes the form

$$
\begin{aligned}
H= & -\mu \sum_{i \sigma} n_{i \sigma}+\sum_{i j \sigma}^{\prime} t_{i j}(n) a_{i \sigma}^{+} a_{j \sigma}+ \\
& +\sum_{i j \sigma}^{\prime}\left(T(i j) a_{i \sigma}^{+} a_{j \sigma} n_{i \bar{\sigma}}+\text { h.c. }\right)+U \sum_{i} n_{i \uparrow} n_{i \downarrow} .
\end{aligned}
$$

As it has been noted, the peculiarity of the model of a material with NEB described by Hamiltonian (2.8) takes into account (as most important) the intersite hoppings of electrons which are caused by the electron-electron interaction and inter-atomic Coulomb and exchange interactions. In this connection the following fact should be noted. Formally, the correlated hopping had been introduced in some papers begining from the pioneer work by S.Schubin and S.Wonsowsky [1]; the possible renormalization of the "band" hopping in the consequence of taking into account the correlated hopping was noted in papers $[5,18,19]$. For the first time, the important role of the correlated hopping in NEB, when the approach was used to obtain splitting into Hubbard subbands, was pointed out in paper [20]. In that work, in particular, it was shown that NEB had an electron-hole asymmetry and essentially renormalized bandwidths connected with the hopping in "hole and doublon subbands". This approach has been developed in papers [21, 22], where it was shown that some properties of the narrow-band materials can be interpreted using the idea of correlated hopping and are caused by the electron-hole asymmetry in NEB.

The fact that taking into account the correlated hopping is of principal necessity was also pointed out in papers [23]. In the recent years models with correlated hopping have been studied intensively [24-28].

\section{Partial cases of the polar model}

\subsection{Weak intra-atomic interaction}

To simplify the consideration we use model Hamiltonian (2.8). If the intraatomic Coulomb interaction is weak $\left(U<\left|t_{i j}(n)\right|\right)$, then we can take into account the electron-electron interaction in the Hartree-Fock approximation:

$$
\begin{aligned}
& n_{i \uparrow} n_{i \downarrow}=n_{\uparrow} n_{i \downarrow}+n_{\downarrow} n_{i \uparrow}, \\
& a_{i \sigma}^{+} n_{i \bar{\sigma}} a_{j \sigma}=n_{\bar{\sigma}} a_{i \sigma}^{+} a_{j \sigma}+\left\langle a_{i \sigma}^{+} a_{j \sigma}\right\rangle n_{i \bar{\sigma}},
\end{aligned}
$$

where the average values $\left\langle n_{i \sigma}\right\rangle=n_{\sigma}$ are independent of site number (we suppose that distributions of the electron charge and magnetic momentum are homogenous). Taking into account (3.1) we can write Hamiltonian (2.8) in the following form:

$$
H=\sum_{i j \sigma}^{\prime} \epsilon_{\sigma}(i j) a_{i \sigma}^{+} a_{j \sigma}
$$

where

$$
\epsilon_{\sigma}(i j)=-\mu+\beta_{\sigma}+n_{\bar{\sigma}} U+t_{i j}(n \sigma) ;
$$




$$
\begin{gathered}
\beta_{\sigma}=\frac{2}{N} \sum_{i j} T(i j)\left\langle a_{i \bar{\sigma}}^{+} a_{j \bar{\sigma}}\right\rangle, \\
t_{i j}(n \sigma)=t_{i j}(n)+2 n_{\bar{\sigma}} T(i j) .
\end{gathered}
$$

The dependences of the effective hopping integral on the electron concentration and magnetization, and the presence of a spin-dependent displasement of the band center are the essential distinctions of the single-particle energy spectrum in the model described by Hamiltonian (3.2) from the spectrum in the Hubbard model for a weak interaction. The use of (3.2) allows one, in particular, to explain the peculiarities of the dependence of the binding energy on the atomic number in transition metals and also essentially modifies the theory of ferromagnetism in a collective electron model.

\subsection{Strong intra-atomic interaction}

For typical narrow-band materials the conditions of a strong $U \gg t(i j)$ or moderate $U \sim t(i j)$ intra-atomic Coulomb repulsion are satisfied. In this case the Hamiltonian (2.6) using the "configurational ideology" of the polar model proposed in [12] can be written in the form suitable for mathematical treatment. Let us rewtrite Hamiltonian (2.6) in "configurational" representations [11,12]. Transition to $\alpha$-operators is given by the formulae:

$$
a_{i \sigma}^{+}=\alpha_{i \sigma}^{+} \alpha_{i 0}-\eta_{\sigma} \alpha_{i 2}^{+} \alpha_{i \bar{\sigma}}, \quad a_{i \sigma}=\alpha_{i 0}^{+} \alpha_{i \sigma}-\eta_{\sigma} \alpha_{i \bar{\sigma}}^{+} \alpha_{i 2},
$$

where $\eta_{\sigma}=+1$ when $\sigma=\uparrow, \eta_{\sigma}=-1$ when $\sigma=\downarrow$, site $i$ cannot be occupied by electron $(|0\rangle)$, singly occupied $(|\sigma\rangle)$ or doubly occupied $(|2\rangle)$. Transition to $X$ operators is given by the formulae:

$$
a_{i \sigma}^{+}=X_{i}^{\sigma 0}-\eta_{\sigma} X_{i}^{2 \bar{\sigma}}, \quad a_{i \sigma}=X_{i}^{0 \sigma}-\eta_{\sigma} X_{i}^{\bar{\sigma} 2}
$$

where $X_{i}^{k l}$ are operators of the transition from state $|l\rangle$ to state $|k\rangle$ on site $i,{ }^{1}$

The Hamiltonian can be written as:

$$
H=H_{0}+H_{1}+H_{1}^{\prime}+H_{e x},
$$

where

$$
\begin{aligned}
H_{0} & =-\mu \sum_{i}\left(X_{i}^{\uparrow}+X_{i}^{\downarrow}+2 X_{i}^{2}\right)+U \sum_{i} X_{i}^{2}+ \\
& +\frac{1}{2} \sum_{i j} V(i j)\left(1-X_{i}^{0}+X_{i}^{2}\right)\left(1-X_{j}^{0}+X_{j}^{2}\right),
\end{aligned}
$$

\footnotetext{
${ }^{1}$ In papers $[11,12]$ the notations of the site transition-operators $B_{k l}^{i}$ were introduced. In this paper we use modern notations $X_{i}^{k l}$ and more convenient notations of state $|i k\rangle$.
} 


$$
\begin{aligned}
& H_{1}=\sum_{i j \sigma}^{\prime} t_{i j}(n) X_{i}^{\sigma 0} X_{j}^{0 \sigma}+\sum_{i j \sigma} \tilde{t}_{i j}(n) X_{i}^{2 \sigma} X_{j}^{\sigma 2}, \\
& H_{1}^{\prime}=\sum_{i j \sigma}^{\prime}\left(t_{i j}^{\prime}(n)\left(X_{i}^{\downarrow 0} X_{j}^{\uparrow 2}-X_{i}^{\uparrow 0} X_{j}^{\downarrow 2}\right)+h . c .\right) \\
& H_{e x}=-\frac{1}{2} \sum_{i j \sigma}^{\prime} J(i j)\left(\left(X_{i}^{\sigma}+X_{i}^{2}\right)\left(X_{j}^{\sigma}+X_{j}^{2}\right)+X_{i}^{\sigma \bar{\sigma}} X_{j}^{\sigma \bar{\sigma}}\right)
\end{aligned}
$$

$X_{i}^{k}$ is the operator of the number of $|k\rangle$-states on site $i$,

$$
\begin{gathered}
\tilde{t}_{i j}(n)=t_{i j}(n)+2 T(i j), \\
t_{i j}^{\prime}(n)=t_{i j}(n)+T(i j) .
\end{gathered}
$$

The expedience of configurational representation is proved by the fact that within it the intraatomic interaction takes a diagonal form. Besides the effects of intraatomic Coulomb interactions, the correlated electron translations are described by Hamiltonians $H_{1}$ and $H_{1}^{\prime}$.

$H_{1}$ describes the transitions from $|j \sigma\rangle$-configurations to $|i 0\rangle$ configurations and from $|j \uparrow \downarrow\rangle$ - configurations to $|j \sigma\rangle$-configurations at neighbour sites, which forms $\sigma-0$ subband ("hole" subband) and $2-$ $\sigma$-subband ("doublon"subband), respectively, (which are analogues to the "lower" and "upper" Hubbard subbands).

$H_{1}^{\prime}$ describes transitions between $\sigma-0$ - and $\uparrow \downarrow-\sigma$-subbands (the processes of the pair creation and destruction of holes and doublons). These processes are "translational" in distinction from the "activational" processes described by $H_{1}$.

If we neglect the inter-atomic Coulomb and exchange interactions in Hamiltonian (3.7), then the Hamiltonian takes the operator structure equivalent to the Hubbard Hamiltonian one. However, in this model the hopping integrals in $\sigma-0-$

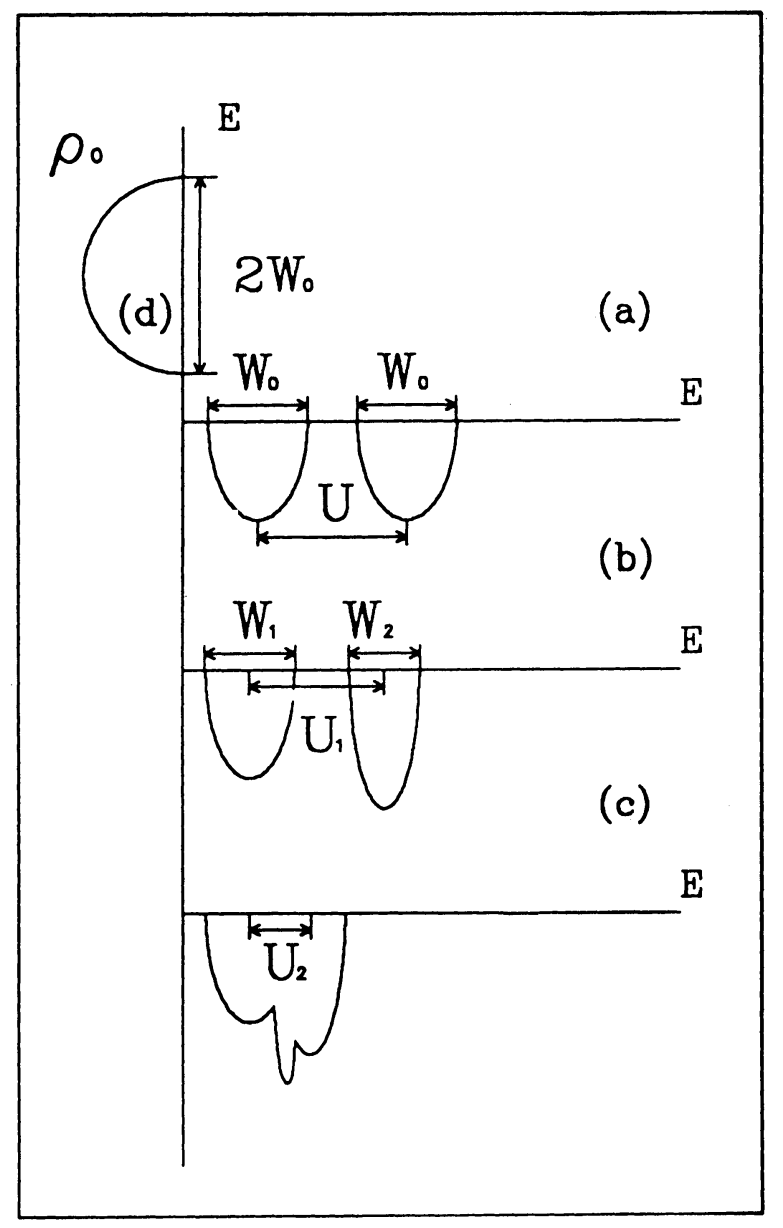

Figure 1. The typical quasiparticle densities of states (a): the Hubbard model; (b), (c): the model with the electron-hole asymmetry ((b) - Mott-Hubbard insulator; (c) - Mott-Hubbard metal); (d): unperturbed band density of states $\rho_{0} ; U, U_{1}, U_{2}$ are the distances between the centers of bands. 
and $\uparrow \downarrow-\sigma$-subbands and "interbands" hopping integrals are concentration dependent and different from the Hubbard model (see figure 1). Properties of this "asymmetrical Hubbard model" can be essentially different.

\subsection{Generalized $t-J$ model}

Configurational representation is especially useful in the investigation of the narrow-band system in which the condition $U \gg t(i j)$ is satisfied. In this case the system can be both a Mott-Hubbard insulator at $n=1$ and a doped Mott-Hubbard insulator at $n \neq 1$. Then the general Hamiltonian, using a suitable form of the perturbation theory [12] and generalizing the Bogolyubov perturbation theory [5] for metallic systems can be written in the form of the effective Hamiltonian which is convenient for mathematical treatment. Thus, the transition to the well-known $t-J$ model occurs (see review [29] and also papers [12, 16] where the modern form of the $t-J$-model was formulated for the first time). Let us use the approach proposed in [12] for the generalized narrow-band Hamiltonian (3.7). Let us perform the canonical transformation

$$
\tilde{H}=e^{s} H e^{-s}
$$

where

$$
S=\sum_{i j}\left(L(i j)\left(X_{i}^{\uparrow 0} X_{j}^{\downarrow 2}-X_{j}^{\downarrow 0} X_{i}^{\uparrow 2}\right)-\text { h.c. }\right) .
$$

If we limit ourselves to the quantities of the second order of a small parameter in equation (3.14) ( $S$ is of the first order), then

$$
\begin{aligned}
\tilde{H} & =H_{0}+H_{1}+H_{1}^{\prime}+\left[S H_{0}\right]+ \\
& +\left[S H_{1}\right]+\left[S H_{1}^{\prime}\right]+\frac{1}{2}\left[S\left[S H_{0}\right]\right] .
\end{aligned}
$$

We use the condition of elimination of "activational" processes

$$
H_{1}^{\prime}+\left[S H_{0}\right]=0
$$

Taking into account the inter-atomic Coulomb interaction in the mean-field approximation we obtain

$$
L(i j)=t_{i j}^{\prime}(n) / \Delta
$$

where

$$
\Delta=U-V+z V\left(\left\langle X_{i}^{0}\right\rangle+\left\langle X_{i}^{2}\right\rangle\right)
$$

is the activation energy of the hole-doublon pair ( $V$ is the strentgh of the Coulomb repulsion between the nearest neighbours). 
The components of commutator $\left[S ; H_{1}\right]$ have an operator structure similar to the one of $H^{\prime}$, but with "hopping integrals" of the second order; in the considered approximation they do not contribute to $\tilde{H}$. Thus, for the case when $\sigma-0$ and $\uparrow \downarrow-\sigma$-subbands are separated by the energy gap and $t_{i}^{\prime} j(n) \ll \Delta$ the initial Hamiltonian (2.6) has the form:

$$
\begin{aligned}
\tilde{H} & =H_{0}+\sum_{i j}^{\prime} t_{i j}(n) X_{i}^{\sigma 0} X_{j}^{0 \sigma}+ \\
& +\sum_{i j \sigma}^{\prime} \tilde{t}_{i j}(n) X_{i}^{2 \sigma} X_{j}^{\sigma 2}+H_{\mathrm{ex}}+\tilde{H}_{\mathrm{ex}}+\tilde{H}_{\mathrm{t}}
\end{aligned}
$$

where

$$
\begin{gathered}
\tilde{H}_{\mathrm{ex}}=-\frac{1}{2} \sum_{i j \sigma}^{\prime} \tilde{J}(i j)\left(X_{i}^{\sigma} X_{j}^{\bar{\sigma}}-\right. \\
\left.-X_{i}^{\sigma \bar{\sigma}} X_{j}^{\bar{\sigma} \sigma}-X_{i}^{2} X_{j}^{0}\right), \\
\tilde{H}_{\mathrm{t}}=-\frac{1}{2} \sum_{i j k \sigma}^{\prime} J(i j k)\left(X_{i}^{\sigma 0} X_{j}^{\bar{\sigma}} X_{k}^{0 \sigma}-X_{i}^{\sigma 0} X_{j}^{\bar{\sigma} \sigma} X_{k}^{0 \bar{\sigma}}\right) \\
-\frac{1}{2} \sum_{i j k \sigma}^{\prime} J(i j k)\left(X_{i}^{2 \sigma} X_{j}^{\sigma \bar{\sigma}} X_{k}^{\bar{\sigma} 2}-X_{i}^{2 \sigma} X_{j}^{\bar{\sigma}} X_{k}^{\sigma 2}\right) .
\end{gathered}
$$

Here

$$
\tilde{J}(i j)=2 t_{i j}^{\prime}(n) t_{i j}^{\prime}(n) / \Delta
$$

is an integral of the indirect exchange (through polar states),

$$
J(i j k)=2 t_{i j}^{\prime}(n) t_{j k}^{\prime}(n) / \Delta
$$

is an integral of the indirect charge transfer in $\sigma$-0- and $\uparrow \downarrow-\sigma$-subbands; in sum (3.22) sites $i$ and $k$ are the nearest neighbours to $j$.

Elimination of the processes of the paired creation and destruction of holes and doublons (in the first order over hopping integral $t_{i j}^{\prime}(n)$ ) leads to the appearance of two terms $\tilde{H}_{\text {ex }}$ and $\tilde{H}_{\mathrm{t}}$ in $\mathrm{EH}(3.20) . \tilde{H}_{\text {ex }}$ describes the indirect exchange interaction (superexchange), $\tilde{H}_{\mathrm{t}}$ describes the indirect hopping of electrons (supperhopping). EH (3.20) generalizes the EH obtained in [12] for the Hubbard model. The distinctions of EH (3.20) from the forms of $t-J$-models $([30,31])$ are caused firstly by the concentration-dependence of the hopping integrals in $\sigma$-0- and $2-\sigma$ subbands, secondly, by the difference of the noted hopping integrals (the absence of the electron-hole symmetry), secondly, and, thirdly, by the unusual form of the superexchange and superhopping integrals (the presence of the concentrationdependence in hopping integrals, formula (3.19) for $\Delta$ ).

In a modified in this way $t-J$-model, in particular, the conditions of the realization of high- $T_{c}$ are more favourable than in the similar Spalek model [32]. The enumerated peculiarities of the model $\mathrm{EH}$ are useful for the interpretation of the physical properties of narrow-band materials. 


\section{4. " $b-c$ "-representation}

We give here another form of the Hamiltonian representation which is useful to study a metal-insulator transition problem. For simplicity we consider the Hubbard Hamiltonian. Let us introduce operators

$$
b_{i \sigma}^{+}=a_{i \sigma}^{+}\left(1-n_{i \bar{\sigma}}\right), \quad c_{i \sigma}^{+}=a_{i \sigma}^{+} n_{i \bar{\sigma}} .
$$

One can see that

$$
a_{i \sigma}^{+}=b_{i \sigma}^{+}+c_{i \sigma}^{+}, \quad a_{i \sigma}=b_{i \sigma}+c_{i \sigma} .
$$

The Hubbard Hamiltonian in this " $b-c$ " representation has the form:

$$
H=H_{0}+H_{1}+H_{1}^{\prime}
$$

with

$$
\begin{aligned}
& H_{0}=-\mu \sum_{i \sigma}\left(b_{i \sigma}^{+} b_{i \sigma}+c_{i \sigma}^{+} c_{i \sigma}\right)+\frac{U}{2} \sum_{i \sigma} c_{i \sigma}^{+} c_{i \sigma}, \\
& H_{1}=t \sum_{i j \sigma}^{\prime}\left(b_{i \sigma}^{+} b_{j \sigma}+c_{i \sigma}^{+} c_{j \sigma}\right) \\
& H_{1}^{\prime}=t \sum_{i j \sigma}^{\prime}\left(b_{i \sigma}^{+} c_{j \sigma}+\text { h.c. }\right),
\end{aligned}
$$

where $t \equiv t_{i j}$. From the operator structures of the latter Hamiltonians one can see that $H_{1}$ describes the translational hopping forming " $b$ " - and " $c$ "-bands and $H_{1}^{\prime}$ describes the "inter-band" hopping. Here one can see a formal analogy between the model described by Hamitontan (3.7) and the "two-configuration" Irkhin model [33].

\section{Single-particle energy spectrum. Metal-insulator transition}

Beyond the frameworks of the approximations considered in Sect. 3 the region of parameters, at which a width of the unperturbed band $2 z|t(i j)|$ and a strength of the Coulomb repulsion are close to each other, is preserved. From general physical considerations we have to expect a metal-insulator transition (we mean $n=1$ ) in this region. Although a great number of papers are devoted to solving the energy gap problem, the question of the correct description of the metal-insulator transition remains in the focus of researchers (see, for example, [34,35]).

The most essential defect of the approximation "Hubbard-I" is the inability to describe the metal-insulator transition (MIT) because of the presence of an energy gap in the spectrum at all values of $U / w>0$. Other approximations are free from this defect, but have their own defects [34,35].

In this paper a new approach to the calculation of the single-particle energy spectrum of narrow-band materials which leads to the correct description of the metal-insulator transition is proposed. This approach is based on a variant of 
the approximate second quantized representation [36] method in the generalized Hartree-Fock approximation (GHFA) [37].

We start from Hamiltonian (3.27) in the " $b-c$ "-representation (3.25). Let us suppose that any kind of electron ordering is absent (in this case the interatomic interaction taken into account in the mean-field approximation leads to the chemical potential renormalization). Let us introduce the Green function:

$$
G_{p s}^{\sigma}(E)=\left\langle\left\langle a_{p \sigma} \mid a_{s \sigma}^{+}\right\rangle\right\rangle .
$$

The single-particle Green function which is written in $b$-c-operators as

$$
G_{p s}^{\uparrow}(E)=\left\langle\left\langle b_{p \uparrow} \mid b_{s \uparrow}^{+}\right\rangle\right\rangle+\left\langle\left\langle b_{p \uparrow} \mid c_{s \uparrow}^{+}\right\rangle\right\rangle+\left\langle\left\langle c_{p \uparrow} \mid b_{s \uparrow}^{+}\right\rangle\right\rangle+\left\langle\left\langle c_{p \uparrow} \mid c_{s \uparrow}^{+}\right\rangle\right\rangle
$$

is given by equation

$$
\begin{aligned}
(E+\mu)\left\langle\left\langle b_{p \uparrow} \mid b_{s \uparrow}^{+}\right\rangle\right\rangle=\quad & \frac{\delta_{p s}}{2 \pi}\left\langle 1-n_{p \downarrow}\right\rangle+\left\langle\left\langle\left[b_{p \uparrow}, H_{1}\right]_{-} \mid b_{s \uparrow}^{+}\right\rangle\right\rangle \\
& +\left\langle\left\langle\left[b_{p \uparrow}, H_{1}^{\prime}\right]_{-} \mid b_{s \uparrow}^{+}\right\rangle\right\rangle,
\end{aligned}
$$

with $[A, B]_{-}=A B-B A$. We suppose in equation (4.3) that

$$
\left[b_{p \uparrow}, H_{1}\right]_{-}=\sum_{j} \epsilon(p j) b_{j \uparrow}, \quad\left[b_{p \uparrow}, H_{1}^{\prime}\right]_{-}=\sum_{j} \epsilon_{1}(p j) c_{j \uparrow}
$$

where $\epsilon(p j)$ and $\epsilon_{1}(p j)$ are non-operator expressions. It gives a closed system of equations for Green functions $\left\langle\left\langle b_{p \uparrow} \mid b_{s \uparrow}^{+}\right\rangle\right\rangle$and $\left\langle\left\langle c_{p \uparrow} \mid c_{s \uparrow}^{+}\right\rangle\right\rangle$. After anticommutation of the both sides of the first formula in (4.4) with $b_{k \uparrow}^{+}$, we have

$$
\begin{aligned}
\epsilon(p k)\left\langle 1-n_{k \downarrow}\right\rangle=\quad & t\left\langle 1-n_{p \downarrow}\right\rangle\left\langle 1-n_{k \downarrow}\right\rangle+t b_{p \downarrow}^{+} b_{p \uparrow} b_{k \uparrow}^{+} b_{k \downarrow} \\
& -\delta_{p k} t \sum_{j} b_{k \uparrow}^{+} b_{j \uparrow}+\delta_{p k} t \sum_{j} c_{j \uparrow}^{+} c_{k \uparrow}+t b_{p \downarrow} c_{p \uparrow} c_{k \uparrow}^{+} b_{k \downarrow}^{+} .
\end{aligned}
$$

The usual method of determining $\epsilon(p k)$ consists in the averaging of expression (4.5). In this way approximations $[3,30,38]$ were obtained; the defects of these approximations are known (see, for example, [39]). Here another approach is proposed (see also [36]).

In (4.5) we write

$$
\begin{gathered}
b_{k \uparrow}^{+}=\alpha_{k \uparrow}^{+} \alpha_{k 0}, \quad b_{k \uparrow}=\alpha_{k 0}^{+} \alpha_{k \uparrow}, \\
c_{k \downarrow}^{+}=-\alpha_{k \downarrow}^{+} \alpha_{k 0}, \quad c_{k \downarrow}=-\alpha_{k 0}^{+} \alpha_{k \downarrow},
\end{gathered}
$$

where $\alpha_{i k}^{+}$is a creation operator of $|k\rangle$-state on $i$-site and $\alpha_{i l}$ is an annihilation operator of $|l\rangle$-state (analogues to the Schubin-Wonsowsky opperators). Let us substitute $\alpha$-operators by $c$-numbers in equation (4.5)

$$
\alpha_{i \sigma}^{+}=\alpha_{i \sigma}=\left(\frac{1-2 d}{2}\right)^{1 / 2}, \quad \alpha_{i 0}^{+}=\alpha_{i 0}=\alpha_{i 2}^{+}=\alpha_{i 2}=d^{1 / 2}
$$


(a non-magnetic case, electron concentration on site $n=1$ ); $d$ is the concentration of the polar states (holes or doublons). From equation (4.5), after transition to $\mathbf{k}$ representation we obtain $\epsilon(\mathbf{k})=(1-2 d) t(\mathbf{k})$. Similarly, we find that $\epsilon_{1}(\mathbf{k})=2 d t(\mathbf{k})$. An analogous procedure is also realized in the equations for other Green functions in (4.2).

Finally, in k-representation the single-particle Green function is

$$
\begin{aligned}
& G_{\mathbf{k}}(E)=\frac{1}{2 \pi}\left(\frac{A_{\mathbf{k}}}{E-E_{1}(\mathbf{k})}+\frac{B_{\mathbf{k}}}{E-E_{2}(\mathbf{k})}\right), \\
& A_{\mathbf{k}}=\frac{1}{2}-\frac{2 d t(\mathbf{k})}{\sqrt{U^{2}+(4 d t(\mathbf{k}))^{2}}}, \quad B_{\mathbf{k}}=\frac{1}{2}+\frac{2 d t(\mathbf{k})}{\sqrt{U^{2}+(4 d t(\mathbf{k}))^{2}}}, \\
& E_{1,2}(\mathbf{k})=(1-2 d) t(\mathbf{k}) \mp \frac{1}{2} \sqrt{U^{2}+(4 d t(\mathbf{k}))^{2}} .
\end{aligned}
$$

The single-particle Green function (4.7) gives exact atomic and band limits: if $U=0$, then $d=1 / 4$ and $G_{\mathbf{k}}(E)$ gets the band form, if $t(\mathbf{k}) \rightarrow 0$, we obtain an exact atomic limit.

The energy gap (difference of energies between the bottom of the upper and the top of the lower Hubbard bands)

$$
\Delta E=-2 w(1-2 d)+\sqrt{U^{2}+(4 d w)^{2}},
$$

$(w=z|t|, z$ is the number of the nearest neighbours to a site).

The difference of equations (4.7), (4.9)-(4.10) from the earlier obtained results (see, for example, $[34,35,40])$ is the dependence of polar states on concentration (and thus on temperature).

At $T=0$ the concentration of polar states is

$$
d=\left(\frac{1}{4}+\frac{U}{32 d w} \ln (1-4 d)\right) \theta(2 w-U)
$$

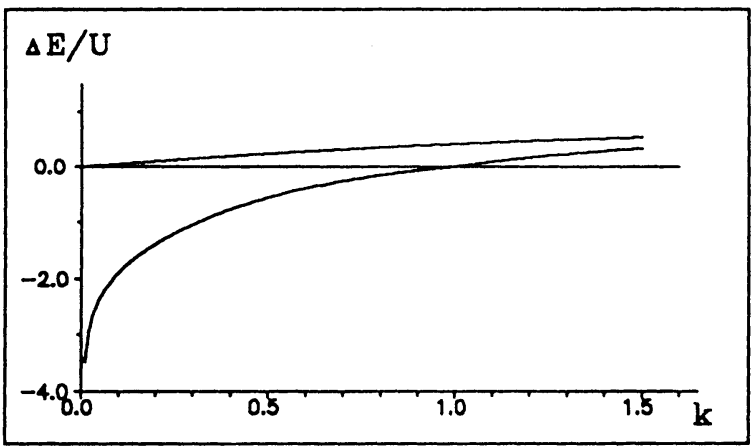

Figure 2. The dependence of the energy gap with $\Delta E$ on parameter $U /(2 \omega)$ in the considered approximation (lower curve) and in the Hubbard-I approximation (upper curve). $(\theta(x)=1$ if $x>0$ and $\theta(x)=0$ if $x<0) . \Delta E \leqslant 0$ when the condition $2 w \geqslant U$ is satisfied (in agreement with general physical ideas [40]). In figure 2 the dependence of the energy gap width on the ratio $U / 2 w$ is shown.

At $T \neq 0$ and given $w, U$, the gap vanishes at $c<c_{0}$, where

$$
c_{0}=\frac{1-(U / 2 w)^{2}}{4} \quad(2 w>U)
$$

if $c>c_{0}$, the insulating state is realized. Thus, the proposed approach allows one to describe a melal-insulator transition. 


\section{Specific narrow-band effects}

\subsection{Absence of electron-hole asymmetry in NEB}

Let us consider the narrow-band system in which the electron concentration $n<1$ and the energy subbands $\sigma-0$ and $\uparrow \downarrow-\sigma$ are separated by gap $\Delta E$. Thus, at temperature $k T \ll \Delta E$ we can limit ourselves to the consideration of the lower $\sigma-0$-subband. The state of such a system (doped Mott-Hubbard insulator DMHI) will be described by EH (3.20) in which we put the tems coresponding to the hopping of $|\uparrow \downarrow\rangle$-states equal to zero.

Now, let us suppose that NEB is in the DMHI state with $n>1$. In the Hubbard model the physical properties of the DMHI system are equivalent both to $n<1$ and to $n>1$ when condition $\left\langle X_{i}^{0}\right\rangle=$ $\left\langle X_{i}^{2}\right\rangle$ is satisfied. This peculiarity of the Hubbard model (doublon-hole or electron- hole symmetry) is the result of the hopping integrals equality in $\sigma-0$ - and $\uparrow \downarrow-\sigma$-subbands. In the proposed model the hopping integrals in both subbands $t_{i j}(n)$ and $\tilde{t}_{i j}(n)$ can be esentially different. Besides, at the transition of the system from the DMHI state with $n<1$

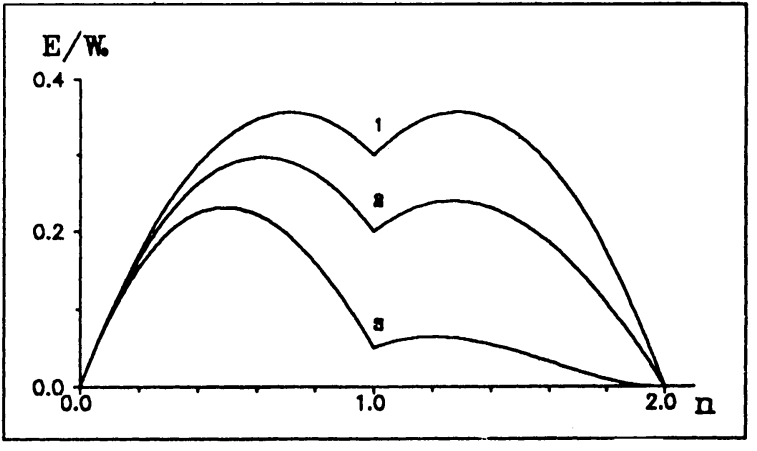

Figure 3. The dependences of binding energy in the considered narrow-band model on filling of the $s$-band; $U / W_{0}=0.8 ; 1$ $-\tau_{1}=\tau_{2}=0 ; 2-\tau_{1}=\tau_{2}=0.1 ; 3-$ $\tau_{1}=\tau_{2}=0.25$. to the DMHI state with $n>1$ the bandwidth has a jump equal to $2 z T(i j)$ (and it continues to decrease with the increase of $n$ in the consequence of taking into account the correlated hopping; see figure 3 ). So the properties of the narrow-band system with a strong intra-atomic interaction can be very different for the cases of $n<1$ and $n>1$ in consequence of the essential difference between subband widths (doublon-hole or electron-hole asymmetry).

This non-equivalence will be shown, in particular, in the dependence of conductivity on the degree of subband filling. In paper [22] it was shown that for the DMHI the conductivity at $n<1$ is $\sigma \sim c n w /(2-n)$, and for $n>1$ it is $\tilde{\sigma} \sim d \tilde{w}(2-n) / n,\left(c=\left\langle X_{i}^{0}\right\rangle, d=\left\langle X_{i}^{2}\right\rangle\right)$. In the region of the electron concentration, for which $\partial \sigma / \partial n>0(n<1)$ and $\partial \tilde{\sigma} / \partial n>0(n>1)$, we have a conductivity of $n$-type, and for $\partial \sigma / \partial n<0$ and $\partial \tilde{\sigma} / \partial n<0$ it is of $p$-type. One can see that the $n-p$-type of conductivity of the narrow-band system in the DMHI state is changed three times with the change of electron concentration from 0 to 2 : in the region of the first and second maximums (if we neglect the correlated hopping, then $n_{1} \simeq 0,6$ and $\left.n_{2} \simeq 1,4\right)$ and at $n=1$. In the region of a certain conductivity type the expressions for the calculation of conductivity can be written in the Drude-Lorentz form with the effective mass depending on the electron concentration [22]. 
The non-equivalence of the cases of $n<1$ and $n>1$ in the concentrationdependence of $\sigma(n)$ is confirmed experimentally. In paper [41] it was shown that in metalooxides with less than a half-filled $3 d$-shell $\left(\mathrm{Mn}_{2} \mathrm{O}\right)$ the conductivity is much higher than in compounds with a half or more than a half-filled $3 d$-shell $(\mathrm{MnO}$, $\mathrm{NiO})$.

\subsection{Application of the model for the consideration of some properties of narrow-band materials}

Let us brieflyly consider the possibility of application of the obtained results for the explanation of some narrow-band system properties.

1. Binding energy of $3 d$-metals. The binding energy in our model is defined (for the case of weak and moderate intra-atomic interactions) by the formula:

$$
E_{b}=-\sum_{k \sigma} \epsilon_{\mathbf{k} \sigma}\left\langle\alpha_{k \sigma}^{+} \alpha_{k \sigma}\right\rangle-\nu U
$$

where $\epsilon_{\mathbf{k} \sigma}$ is the Fourier-component of $t_{i j}, \nu=n^{2} / 4$ for $n<1$ and $\nu=1-n+n^{2} / 4$ for $n>1$. In the approximation of the rectangular density of states the binding energy has the form:

$$
E_{b}=\frac{1}{2 w(n)}\left[w^{2}(n)-t_{c}^{2}\right]-\nu U
$$

with

$$
w(n)=w_{0}\left[1-n\left(\tau_{1}+\tau_{2}\right)\right], \quad t_{c}=w(n)[n-1],
$$

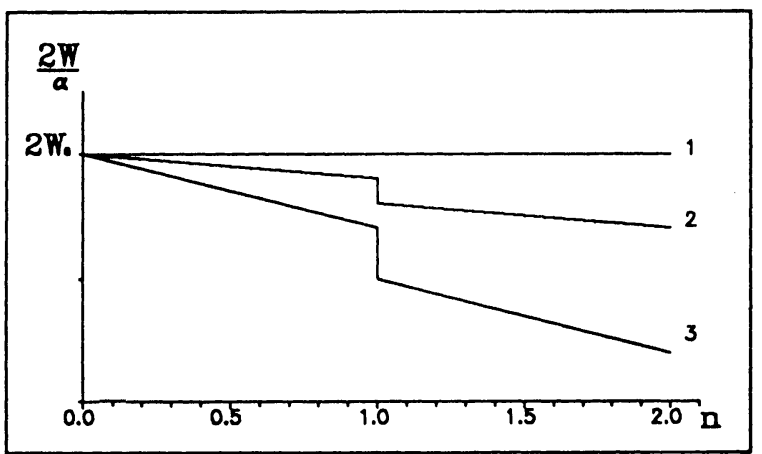

Figure 4. The dependences of the normalized bandwidth at transition from the hole $(n<1)$ to the electron $(n>1)$ type of conductivity in the doped Mott-Hubbard insulator. 1 corresponds to the Hubbard model $\left(\tau_{1}=\tau_{2}=0\right) ; 2-\tau_{1}=\tau_{2}=0.1 ; 3-$ $\tau_{1}=0.35, \tau_{2}=0.25$. where $\tau_{1}, \tau_{2}$ are parameters of the correlated hopping, $2 w_{0}$ is the unperturbed bandwidth. The dependence of the binding energy on the $d$ electron concentration in $3 d$-systems can be determined by the generalization of equation (5.1) for the case of five-equivalent $d$-subbands. Figure 4 shows that the obtained results explain the peculiarities of the dependence of binding energy on the atomic number: minimum for $\mathrm{Mn}$ and the presence of two nonequivalent maximums ( $\mathrm{V}, \mathrm{Co}$ ) (as a result of taking into account the correlated hopping).

2. MIT under the action of external influences. From equation (4.20) 
one can see that the energy gap increases with the increase of the current carrier concentration at given $U$ and $w$ (i.e. at a constant external pressure). This increase can be caused by the increase of temperature; the condition of the metallic state realization $c<c_{0}$ in this case cannot be satisfied. The obtained temperature dependence of $\Delta E$ can explain the observable transition in $\left(\mathrm{V}_{1-x} \mathrm{Cr}_{x}\right)_{2} \mathrm{O}_{3}$ [42] and $\mathrm{NiS}_{2}$ [43] from a paramagnetic metal to a Mott-Hubbard insulator with the increase of temperature.

The dependence of $\Delta E$ on the polar state concentration points out to the possibility of specific narrow-band effects giving an opportunity to control MIT with the help of a magnetic field and photoeffect. For example, a strong magnetic field can cause a decrease of the polar state concentration $d$ [22] and, therefore, MIT occurs. Otherwise, the increase of $d$ caused by photoeffect will stimulate the reverse metal-to-insulator transition (analogous to the temperature change).

3. Change of the $n-p$ type of conductivity. The change of the conductivity type about half-filling noted in Sect. 4 is confirmed experimentally for some compounds, e.g. $\mathrm{VO}_{x}$; here, within the framework of the considered model MottHubbard insulator, the state at $\mathrm{x}=0$ corresponds to the electron concentration $n=1$ (modelling half-filled $t_{2} q$-band). At $\mathrm{x}>1$ in $\mathrm{VO}_{x}$ the holes $\left(\mathrm{V}^{3+}\right)$ appear, whereas at $x<1$ the doublons $\left(\mathrm{V}^{+}\right)$appear. In accordance with the result of Sect. 4, experiment [40] exhibits at $\mathrm{x} \simeq 1$ a transition from the $p$-type $($ at $\mathrm{x}>1$ ) to $n$-type conductivity (at $x<1$ ). The analogous change of the conductivity type is also observed in $\mathrm{Co}_{x} \mathrm{Fe}_{3-x} \mathrm{O}_{3}$ [44].

4. Concentration dependence of the activation energy. In consequence of the concentration dependence of the parameters which determine the quasiparticle energy spectrum in $\sigma-0$ and $\uparrow \downarrow-\sigma$-subbands, the activation energy has a jump at $n=1$ at the transition of the system from the state with $n<1$ to the state with $n>$

1. In this case both the increase and decrease of the activation energy are possible depending on the mutual arrangement of the $\sigma-0$ and $\uparrow \downarrow-\sigma$-subbands relatively to other bands. This jump of the activation energy is confirmed experimentally for $\mathrm{Mn}_{x} \mathrm{Fe}_{3-x} \mathrm{O}_{4}$ [44] and $\mathrm{Co}_{x} \mathrm{Fe}_{3-x} \mathrm{O}_{4}$ [41].

\section{Acknowledgment}

Here I would like to express my sincere gratitude to Prof. I.Stasyuk for our long lasting fruitful collaboration and for his permanent interest in my research.

\section{References}

1. Schubin S., Wonsowsky S. On the electron theory of metals. // Proc. Roy. Soc. A, 1934, vol. 145, p. 159-180.

2. Samsonov V.G., Priadko L.Ph., Priadko I.Ph. Electron localization in solid. Moscow, Nauka, 1976 (in Russian).

3. Hubbard J. Electron correlation in narrow energy bands. // Proc. Roy. Soc. A, 1963, vol. 276, No 1365, p. 238-257. 
4. Samojlovich A.G., Kondratenko V.M. On atomic semiconductor theory. // Ukr. Fiz. Zhurn., 1958, vol. 3, No 1, p. 40-51 (in Ukrainian).

5. Bogolyubov M.M. Lectures on quantum statistics. Kyiv, Radianska Shkola, 1948 (in Ukrainian).

6. Wonsowsky S., Karpenko B. Handbuch der Physik. Bd.18/1. Berlin, Springer, 1968 (in German).

7. Glauberman A.Yu., Vladimirov V.V., Stasyuk I.V. New form of the polar model of crystal. // DAN USSR, 1959, vol. 126, No 3, p. 543-545 (in Russian).

8. Glauberman A.Yu., Vladimirov V.V., Stasyuk I.V. Theory of atomic excitations in atomic crystals. // Fiz. Tverdogo Tela, 1960, vol. 2, p. 133-143 (in Russian).

9. Stasyuk I.V. Approximate second quantized method in the many-electron theory of non-metallic crystals. // Visnyk Lvivskoho Universytetu, phys. ser. 1962, vol. 1, p. 3244 (in Ukrainian).

10. Hubbard J. Electron correlation in narrow energy bands. The atomic representation. // Proc. Roy. Soc. A, 1965, vol. 285, No 1403, p. 542-560.

11. Didukh L.D., Stasyuk I.V. Effective Hamiltonian in the Anderson model. // Fiz. Met. Metalov., 1968, vol. 26, No 4, p. 582-588 (in Russian).

12. Didukh L.D., Stasyuk I.V. On ferromagnetism theory in the polar model. // Ukr. Fiz. Zhurn., 1968, vol. 13, No 6, p. 899-904 (in Russian).

13. Didukh L.D., Priadko L.Ph., Stasyuk I.V. Correlation effects in materials with narrow energy bands. Lviv, Vyshcha shkola, 1978 (in Russian).

14. Didukh L. A model of the narrow-band material with the electron-hole assymetry. // J. Phys. Studies, 1997, vol. 1, No 2, p. 241-250 (in Ukrainian).

15. Didukh L.D. Some properties of materials with narrow energy bands. // UkrainianFrench Simposium "Condensed Matter: Science and Industry". Abstracts, p. 275 (Lviv, 1993).

16. Didukh L.D., Stasyuk I.V. On ground state energy and ferromagnetism condition in narrow s-band. // Fiz. Met. Metalov., 1972, vol. 33, p. 429-431 (in Russian).

17. Didukh L.D. On ferromagnetism theory in narrow energy bands // Ukr. Fiz. Zhurn., 1988, vol. 33, No 3, p. 449-454 (in Russian).

18. Wonsowsky S.V., Svirsky M.S. On statistics of spin and polar excitations in crystals. // Zh. Exp. Theor. Phys., 1958, vol. 35, No 6, p. 1447-1454 (in Russian).

19. Caron L.G., Pratt G.W. Correlation and magnetic effects in narrow energy bands. // Rev.Mod.Phys., 1968, vol. 40, No 4, p. 802-806.

20. Didukh L.D. On taking into account correlation effects in narrow energy bands. // Fiz. Tverd. Tela, 1977, vol. 19, p. 1217-1222 (in Russian).

21. Didukh L.D. Ordered states in materials with narrow energy bands. In: Mechanizm of two-electron dynamics of electrons in non-organic materials. Moscow, 1990, p. 166-186 (in Russian).

22. Didukh L. Correlation effects in materials with non-equivalent Hubbard subbands. Preprint of Institute for Condensed Matter Physics, ICMP-92-9P, Lviv, 1992, p. 32 (in Russian).

23. Hirsh J.E. // Phys. Rev. B, 1984, vol. 163, p. 11515; Hirsh J.E. // Physica B, 1990, vol. 163, p. 291; Hirsh J.E. // Physica B, 1994, vol. 199-200, p. 366.

24. Lin H.Q., Hirsch J.E. Pairing in a tight-binding model with occupation-dependent hopping rate: Exact diagonalization study. // Phys. Rev. B, 1995, vol. 52, No 22, p. 16155-16164; Amadon J.C. and Hirsch J.E. Mettalic ferromagnetism in a single- 
band model: Effect of band filling and Coulomb interactions. // Phys. Rev. B, 1996, vol. 54, No 9, p. 6364-6375.

25. Schadschneider A. Superconductivity in an exactly solvable Hubbard model with bond-charge interaction. // Phys. Rev. B, 1995, vol. 51, No 16, p. 10386-10391.

26. Gagliano E.R., Aligia A.A., Arrachea L., Avignon M. Single-particle spectral function of a generalized Hubbard model: Metal-insulator transition. // Phys. Rev. B, 1995, vol. 51, No 20, p. 14012-14019; Gagliano E.R., Aligia A.A., Arrachea L., and Avignon M. Electronic properties of a generalized Hubbard model at half-filling. // Physica B, 1996, vol. 223-224, No 1-4, p. 605-607; Arrachea L., Gagliano E.R., and Aligia A.A. Ground-state phase diagram of an extended Hubbard chain with correlated hopping at half-filling. // Phys. Rev. B, 1997, vol. 55, No 2, p. 1173-1184.

27. Santoro G., Manini N., Parola A., and Tossatti E. Phase diagram of a model of correlated hopping of electrons in a lattice of Berry molecules. // Phys. Rev. B, 1996, vol. 53, No 1, p. 828.

28. Chattopadhyay B. and Gaitonde D.M. Phase diagram of the half-filled extended Hubbard model in two dimensions. // Phys. Rev. B, 1997, vol. 55, No 23, p. 15364-15367.

29. Izyumov Yu.A. Strongly correlated electrons: $t-J$-model. // Usp. Fiz. Nauk, 1997, vol. 167, No 5, p. 465-497 (in Russian).

30. Harris A.B. and Lange V. Single-particle excitations in narrow energy bands. // Phys. Rev. B, 1967, vol. 157, No 2, p. 295-314.

31. Chao K.A., Spalek J., Oles A. The kinetic exange interaction in doubly degenerate narrow band. // Phys. Stat. Sol. (B), 1977, vol. 84, p. 747-759; Chao K.A., Spalek J., Oles A. // J. Phys. C., 1977 vol. 10, p. L271.

32. Spalek J. Statistical properties of the spin liquid. // Phys. Rev. B, 1988, vol. 37, No 1 p. $535-536$.

33. Irkhin Yu.P. Two-configurational Hubbad model. // Fiz. Tverdogo Tela, 1993, vol. 35, No 6, p. 1432-1442 (in Russian).

34. Izyumov Yu.A. The Hubbard model in the regime of strong electronic correlations. // Usp. Fiz. Nauk., 1995, vol. 165, No 4, p. 403-427 (in Russian).

35. Gebhard F., The Mott Metal-Insulator Transition - Models and Methods, Berlin, Springer, 1997.

36. Didukh L. Energy spectrum of electrons in the Hubbard model: a new mean-field approximation. // Phys. Stat. Sol. (B), 1998, vol. 206, p. R5-R6.

37. Roth L. // Phys. Rev. Lett., 1968, vol. 20, p. 1431 ; Zubarev D.N., Rudoy Yu.G. On calculation of correlation functions in quantum statistical physics. // Usp. Fiz. Nauk, 1993, vol. 163, No 3, p. 103-106 (in Russian).

38. Roth L. Electron correlations in narrow energy bands. // Phys.Rev., 1969, vol. 184, No 2, p. 451-459.

39. Kawabata A. One electron Green's function in magnetic insulators. // Progr. Theor. Phys., 1972, vol. 48, p. 1793-1808.

40. Mott N.F. Metal-Insulator Transitions. London, Taylor and Francis, 1990.

41. Jonker G.H. // Journ. Phys. Chem. Solids., 1959, vol. 9, p. 165.

42. Mc Whan D.B., Remeika J.P. Metal-insulator transition in metaloxides. // Phys. Rev. B, 1970, vol. 2, p. 3734-3739.

43. Wilson J.A., Pitt G.D.. Metal-insulator transition in $\mathrm{NiS}_{2}$ Phil.Mag., 1971, vol. 23, p. $1275-1296$.

44. Krupichka S. Fizika ferritov, vol.2. Moscow, Mir, 1976 (in Russian). 


\section{Модифікована форма полярної моделі кристалів}

\section{Л.Дідух}

Тернопільський державний технічний університет імені І. Пулюя, кафедра фізики, 282001 м. Тернопіль, вул. Руська, 56

Отримано 22 травня 1998 р.

Запропонована модель вузькозонного матеріалу, особливістю якої $\epsilon$ врахування корельованого переносу електронів (зумовленого електрон-електронною взаємодією). У даній моделі має місце електронно-діркова асиметрія (на противагу електронно-дірковій симетрії, яка характерна для моделі Хаббарда). Для випадків слабких та сильних внутрішньоатомних взаємодій гамільтоніан моделі представлений у формі ефективного гамільтоніана, який узагальнює відомі форми ефективних гамільтоніанів. Досліджений перехід метал-діелектрик. Розглянуто застосування запропонованої моделі до пояснення особливостей фізичних властивостей систем з вузькими зонами провідності.

Ключові слова: вузькі зони провідності, модельний гамільтоніан, корельований перенос, електрон-діркова асиметрія

PACS: $71.28 .+d, 71.27 .+a, 71.10 . F d, 71.30 .+h$ 
